\title{
Antimalarials - old drugs are new again
}

\author{
Anna Woźniacka \\ Department of Dermatology and Venereology, Medical University of Lodz, Lodz, Poland
}

Adv Dermatol Allergol 2022; XXXIX (2): 239-244

DOI: https://doi.org/10.5114/ada.2021.108423

\begin{abstract}
The aim of this study was to discuss the therapeutic mechanism of action of antimalarials, in particular hydroxychloroquine (HCQ), in patients with lupus erythematosus. It examines the results of recent studies indicating the need to revise the indications, dosage and duration of safe therapy, thus limiting the possibility of adverse effects. Historically, HCQ was recommended for patients with arthritis as a predominant symptom, whereas today, it is recommended for all lupus patients, even those with lupus nephritis and pregnant women; it has a favourable therapeutic profile, particularly regarding its effect on the activity of the disease, and the possibility of prolonging survival time and preventing organ damage and dysfunction. Antimalarials are basic, disease-modifying antirheumatic drugs, which not only relieve the symptoms of inflammation, but also affect the underlying processes; therefore, they should be administered chronically, and the expected effects may sometimes take a long time to become apparent. In the past, the recommended dose was $6.5 \mathrm{mg} / \mathrm{kg}$ ideal body weight, whereas today it is up to $5.0 \mathrm{mg} / \mathrm{kg}$ real body weight. A very important aspect of long-term therapy is the strict adherence to the medical prescription; hence, the introduction of a new formulation containing $400 \mathrm{mg}$ of hydroxychloroquine sulphate per tablet is an interesting proposal.
\end{abstract}

Key words: antimalarials, chloroquine, hydroxychloroquine, lupus erythematosus.

\section{Introduction}

Antimalarial preparations have been widely used for many years. However, the results of contemporary laboratory, experimental and clinical studies have yielded new information regarding their use which can raise new hope.

The history of antimalarial drugs dates back to the $17^{\text {th }}$ century. According to the legend, in 1630, Ana de Chinchon, wife of the Viceroy of Peru, was cured of malaria by the indigenous peoples with an extract made from the bark of the "fever tree" [1], which was soon honoured with the name Cinchona tree. Following the treatment, the alkaloid derived from its bark was imported to Europe and became the focus of Western medicine. The bark contains more than 25 different alkaloids, only some of which have been used in medicine. Of these, quinine is one of the best known and was originally used as an antimalarial and antipyretic. The first descriptions of its effective action against lupus were recorded in 1894 by Payne [2]. Later, during World War II, the synthetic derivative of quinine, quinacrine, was widely used to prevent malaria in soldiers fighting in Asia.
Over the years, the number of indications for its use have expanded, and so have its side effects. As such, there has been a need for new, synthetic compounds with a more favourable therapeutic profile. At present, the most commonly used ones are chloroquine (CQ) and hydroxychloroquine (HCQ), which differs from the former by one hydroxyl group. The two preparations are characterized by similar pharmacokinetics, metabolism and mechanism of action [3]. Based on 60 years of research and therapy, it is widely agreed that the widespread use of antimalarial drugs in patients with various connective tissue diseases, particularly lupus erythematosus is recommended: they demonstrate high safety and effectiveness and, not without significance, low treatment costs [4].

\section{Mechanism of action}

The mechanism of action of antimalarial drugs is complex and still not fully understood. CQ and HCQ are weak bases that readily cross phospholipid cell membranes and accumulate within lysosomes, thus increasing the $\mathrm{pH}$ from the initial 4.0 to about 6.0 [3]. The change in $\mathrm{pH}$ contributes to the stabilization of lysosom-

Address for correspondence: Prof. Anna Woźniacka PhD, MD, Department of Dermatology and Venereology, Medical University of Lodz, Plac Hallera 1 building, 90-647 Lodz, Poland, phone: +48 4263930 93, e-mail: wozniacka@bmp.net.pl

Received: 25.03.2021, accepted: 30.04.2021. 
al cell membranes and may thus protect keratinocytes from destruction caused by the action of lysosomal proteases, which are known to be present at high levels in lupus patients. Moreover, the vacuolization of lysosomes contributes to the dysfunction of immunocompetent cells and inhibits the process of antigen presentation.

One of the fundamental causes of systemic lupus erythematosus (SLE) is the dysfunction of the immune system resulting in a loss of tolerance toward autoantigens and the inability to recognize them [4]. This results in the increased production of autoantibodies, the formation and deposition of immune complexes in tissues and the subsequent activation of the pro-inflammatory cytokine system, leading to tissue destruction and the development of organ failure. The major steps in this pathological cascade are currently considered to be loss of immune tolerance, increased antigen exposure, excessive Th cell activity, defective B cell suppression and abnormal $T$ cell response, followed by dysfunction of phagocytic cell function, and the development of inflammation mediated by pro-inflammatory cytokines [5]. Antimalarial preparations inhibit the production of pro-inflammatory cytokines, mainly interleukin (IL) $1 \beta$, IL- 6 and tumor necrosis factor- $\alpha$ (TNF- $\alpha)$ [6].

Within the macromolecular protein complexes inflammasomes are located in the cytoplasm. These act as part of the innate immune response, and their activation enables the maturation and secretion of pro-inflammatory cytokines, including IL-1 and IL-18. However, over-activation of the NRLP3 inflammasome plays an important role in the pathogenesis of many inflammatory diseases, including systemic lupus erythematosus (SLE). It has been reported that HCQ can play a valuable role in SLE treatment by inhibiting inflammasome activity [7]: selective inhibition of the NLRP3 inflammasome offers greater therapeutic benefits than blocking the action of the cytokines further down the signalling pathway.

SLE patients also have increased serum interferon $\alpha$ (IFN- $\alpha$ ) concentrations, which correlate with both the activity and severity of the disease process [8]. IFN- $\alpha$ stimulates apoptosis and cell breakdown, which contributes to the accumulation of material from the cell nuclei [9]. Until recently, it was thought that broken cells were mainly removed by phagocytosis; however, recent studies indicate a new, independent mechanism involving extracellular traps, i.e. network-like structures formed by neutrophils (neutrophil extracellular traps - NETs), created by the release of cell nucleus contents into the extracellular space, together with their granularity components. NET traps are composed of DNA, histones and cytoplasmic granules. They have the ability to stimulate toll-like receptors (TLRs), guardians of innate immunity. The nucleic acid fragments are recognised as foreign antigens by TLR7 and TLR9, which activate the mechanisms of innate immunity through the production of pro-inflammatory cytokines, especially IFN- $\alpha$ and IL-6 [10, 11]. TLR activation plays a key role in SLE, and their inhibition by HCQ effectively inhibits the inflammatory response [12]

The accumulation of HCQ in the lysosomes of dendritic cells, macrophages and B lymphocytes inhibits the process of antigen presentation to $\mathrm{T}$ lymphocytes with the participation of MHC, i.e. major histocompatibility complex, molecules [13].

The mechanism behind the photoprotective action of antimalarial drugs has not been fully understood so far. Currently, it is believed that it is associated with their inhibitory effect on the mediators of the inflammatory process, mainly cytokines and prostaglandins [14]. It has also been proposed that the drug forms deposits in the skin that absorb ultraviolet radiation [15]. The photoprotective effect of hydroxychloroquine sulphate has been used not only in the treatment of connective tissue diseases, but also in the prevention of polymorphic light eruption. While individual reports of photoallergic reactions have appeared in the literature, they seem to be associated with individual hypersensitivity rather than with the mechanism of action of the drug.

\section{Antimalarials in SLE patients with cardiovascular diseases}

SLE patients have a two or even threefold greater risk of premature death [16], with cardiovascular diseases being among the leading causes of death [17]. In SLE patients, immune system dysfunction appears to promote atherogenesis. One of its first signs is dysfunction of endothelial cells, which manifests itself through increased expression of adhesive particles and impaired vasodilation. Endothelial cell damage is affected by an accumulation of reactive oxygen species (ROS) and a decreased antioxidant defence. Several clinical studies have shown a positive correlation between the activity of the disease process in lupus and oxidative stress, i.e. the imbalance between the activities of ROS and the antioxidative system. Endothelial cell damage is also associated with abnormalities in the lipid profile and the presence of anti-phospholipid antibodies, increased activation of monocytes and hyper-expression of TLRs.

Clinical and experimental studies on animals have shown that both CQ and HCQ contribute to a reduction in traditional atherogenic factors, such as lipids and glucose [18-20]. However, it remains undecided whether drugs from this group reduce the risk of developing cardiovascular diseases. Some authors indicate that they contribute to a significant reduction [21-24], while others indicate a neutral effect [25]. A meta-analysis of 19 observational studies including 19,679 patients treated with CQ/HCQ and untreated controls identified a 30\% reduction in the risk of developing cardiovascular diseases, mainly by reducing blood pressure, glucose, cholesterol, triglycerides and low-density lipoprotein levels; the latter contributes to the deposition of a choles- 
terol molecule in the smooth muscle fibres of the arterial walls [26]. These findings indicate that antimalarial drugs significantly reduce the risk of developing cardiovascular diseases [26].

\section{Antimalarials in lupus patients}

Clinical studies show that the course of SLE differs between individuals [27]. In the majority of patients, i.e. about $70 \%$, the disease is chronic, with subsequent periods of exacerbations and remissions. It is estimated that long-term remissions persist for many years in approximately $10 \%$ of patients, the chronic active phase is maintained in another $10 \%$, and the course is mixed in approximately $10 \%$ of patients [27]. Longer periods of remission obviously offer a more favourable prognosis for the patients. The activity of the disease process and the course of SLE are influenced by various factors, such as genetic predisposition, turbulent and multi-symptomatic onset of the disease, lifestyle, early treatment, systematic therapy and, most importantly, compliance with medical recommendations. Many studies show that the administration of CQ and HCQ has a preventive effect, delays the development of systemic lupus symptoms, reduces the number of exacerbations and alleviates its course [28].

In recent years, a low rate of the drug prescription and non-compliance with medical recommendations have become significant medical problems. Regarding the former, views on the importance of antimalarials, especially HCQ, in the treatment of SLE have changed significantly over the past decade. Only a few years ago, the EULAR recommendations advised HCQ for patients whose dominant symptom is arthritis, without any life-threating organ dysfunctions; however nowadays, the drug is considered basic and recommended to all lupus patients, even patients with lupus nephritis and pregnant women. This has been attributed to its favourable therapeutic profile, especially its impact on the disease process, the possibility of extending the survival time and preventative effect on the development of organ damage [28].

Non-compliance with medical recommendations is commonly observed among young, black or poorly-educated people with a low level of general medical knowledge. A meta-analysis by Garg et al. [29] found routine measurement of HCQ concentration in the blood of patients to be an effective method of monitoring compliance. Concentrations equal to and higher than $750 \mathrm{ng} / \mathrm{ml}$ indicate the correct therapeutic dose, which correlates inversely with the activity of the disease process assessed on the SLEDAI scale. Doses lower than $200 \mathrm{ng} / \mathrm{ml}$ indicate a definite lack of compliance and an increased risk of disease activity. Although drug concentration monitoring is of great clinical importance, a significant practical limitation is the high cost of the test [29].

Mehat et al. [30] report that between $43 \%$ and $75 \%$ of SLE patients do not follow medical recommendations and
$33 \%$ make an arbitrary decision to discontinue treatment after 5 years. Discontinuation may be due to the fact that it is an uncomfortable form of therapy. Even so, the number of tablets taken per day also appears to influence the decision; as such, tablets containing $400 \mathrm{mg}$ of hydroxychloroquine sulphate seem to be more convenient and may improve compliance, especially in the initial period of therapy, when the doses of the drug are higher.

\section{Therapeutic doses}

There is some controversy in the literature regarding the recommended doses of HCQ. Doses below $6.5 \mathrm{mg} /$ $\mathrm{kg}$ ideal body weight were considered safe, i.e. low-risk, provided that they are converted into correct, i.e. heightappropriate, body weight. This view was developed on the basis of clinical trials confirming a low accumulation of the drug in adipose tissue. However, the preparation was often overprescribed in obese people, with a greater risk of side effects. Hence, in recent years, a dose of $5.0 \mathrm{mg} / \mathrm{kg}$ real body weight/day based on the actual patient weight has been preferred.

A large study revealed that toxicity is not rare among long-term users of the drug and the risk is highly dependent on the daily dose according to body weight. At a dose $\leq 5 \mathrm{mg} / \mathrm{kg}$ real weight, the risk of incorrect conversion and the development of side effects, especially ophthalmic toxicity, is low [31]. Ophthalmologic toxicity is of serious concern because it is not treatable; therefore, the aim of screening for retinopathy is not to stop valuable drugs at the first borderline abnormality, but to recognize definitive signs of toxicity at an early enough stage to prevent a loss of visual acuity. It is now believed that eye check-ups should be performed prior to treatment or in the first weeks to rule out pre-existing maculopathy. In patients treated with doses $\leq 5 \mathrm{mg} / \mathrm{kg}$ real body weight and without major risk factors, later annual assessments may be performed after 5 years of treatment. When administering the drug at the preferred dose, in the first 5 years of therapy, the risk of developing side effects on the eye appears to be negligible [32]. Patients with preexisting ophthalmic disorders or other risk factors such as age over 65 , renal failure, hypertension may require more careful eye monitoring. In the event of visual disturbances, such as deterioration of visual acuity or colour vision, use should be discontinued immediately and the patient should undergo immediate ophthalmological inspection and close monitoring.

Observational studies conducted for over 30 years indicate that in smokers, antimalarial drugs are not only less effective, but also are more likely to cause adverse reactions, especially in the eyes. Moreover, this group of patients are more likely to demonstrate more intense disease activity [33]. Hence, there is a need to inform patients of the risk and encourage them to quit smoking. It should be remembered that smokers often demonstrate 
a less healthy general lifestyle, placing a lower emphasis on eating healthily, excessive alcohol consumption, high-risk behaviours and lower compliance with medical recommendations [33].

One group of frequently-reported adverse reactions are digestive tract ailments such as indigestion, stomach cramps, nausea, vomiting and diarrhoea; however, these occur significantly less frequently in patients treated with HCQ than CQ [34]. These symptoms can be reduced or eliminated by lowering the dose of the drug, or by using the preparation after a meal or together with products such as antacids or carbon which have a local action in the gastrointestinal tract.

It should be remembered that glucose levels may drop following treatment with antimalarial preparations, and especially in patients simultaneously receiving antidiabetic drugs. Moreover, in some patients, HCQ can prolong the QTc interval. The risk is increased with congenital or acquired QT prolongation, heart failure or recent myocardial infarction, bradycardia, ventricular arrhythmia, hypokalaemia and/or hypomagnesaemia, concomitant use of citalopram, escitalopram, hydroxyzine, domperidone. Simultaneous use of cyclosporine may increase its concentration in the blood, and increase creatinine levels. People treated with thyroid hormones are at a risk of developing clinical hypothyroidism [35].

The drugs from this group are often referred to as basic or disease-modifying antirheumatic drugs (DMARDs), which means that they have the ability to prevent destructive changes in the course of rheumatic diseases, such as rheumatoid arthritis or SLE. Basic medications not only alleviate the symptoms of inflammation, but more importantly, affect the underlying processes. Hence, they are usually administered chronically, until the risk of their administration outweighs the clinical effects of the therapy.

\section{Hydroxychloroquine and pregnancy}

Research work carried out over the last decades indicates that HCQ is a good treatment option for many connective tissue diseases. Clinicians are always concerned about administering drugs to pregnant women. Although a few systematic clinical trials have been conducted, a study has been performed on 33 women with SLE who received HCQ during their 36 pregnancies, and 53 women with the same disease who did not [36]. Both disease activity and gynaecological aspects were assessed, i.e. the course of pregnancy and childbirth, and the clinical condition of the newborn children. Pregnancy was successful in $86 \%$ of patients treated with HCQ and $83 \%$ in the control group. Preterm delivery, i.e. before 37 weeks, was reported in 55\% of treated women and $48 \%$ in the control group. No other statistically significant differences were observed between the populations regarding intrauterine development of the foetus, method of delivery termination or newborn weight. No birth defects were observed. In 2 patients, an increase in lupus activity was observed, but this was effectively controlled by adding HCQ (200 mg/day) or increasing the dose up to $400 \mathrm{mg} /$ day. In addition, the group of pregnant women treated with HCQ received lower doses of concurrently administered prednisolone. The authors report that HCQ administration appears safe during pregnancy, and that disease intensification was observed when the drug was discontinued due to pregnancy. They also emphasize its anticoagulant properties, which enables effective prevention of blood clot development and the possibility to reduce the dose of simultaneously administered corticosteroid drugs (steroid sparing effect).

It should also be remembered that HCQ accumulates in tissues, particularly in the liver and in structures containing melanocytes, i.e. in the skin and the retina of the eye. Therefore, discontinuation of the drug does not eliminate the drug immediately. It has been found to remain in the liver for another 8 weeks and in the skin for more than 6 months [37]. Hence, discontinuation of therapy when pregnancy is diagnosed does not prevent drug exposure, especially in the first trimester, which is crucial for organogenesis. This fact additionally supports the safety of the drug in pregnant women.

HCQ has also been found to play a protective role against the development of cardiac lesions in children born with neonatal lupus erythematosus [38].

\section{Hydroxychloroquine, SLE and COVID-19}

Antimalarials, particularly HCQ, are used widely for the treatment of SLE, rheumatoid arthritis and related inflammatory and dermatologic conditions. These drugs are now being considered for a new application in diabetes mellitus, heart disease, and adjunct cancer therapy; they show a wide spectrum of anti-inflammatory activity, and have been tested against various protozoal bacterial, fungal and viral infections. In the 1990s, due to their good tolerance, low risk of toxicity, favourable immunomodulating effect and low production cost, they were evaluated for use against HIV infection. Administration was found to slow the HIV replication process, which seemed to be a good basis for its use in other viral diseases such as Ebola, influenza, MERS, SARS or SARSCoV-2, the cause of the current pandemic [39].

A pandemic disrupts the functioning of many areas of social life, especially health care, with the emergence of new threats, questions and doubts. Over the past year, studies have examined the impact of immunosuppressive treatment on the risk of developing the infection caused by the SARS-CoV-2 virus in patients with various connective tissue diseases. The results appear inconclusive, although patients have comorbidities and immunosuppressive treatment could increase the risk of infection. The study was performed in Milan, Italy during 
the most difficult period of the pandemic, i.e. between February and April 2020. The results do not suggest that this group is at an increased risk of infection compared to the general population [40]. Moreover, none of the 955 patients demonstrated a severe course of COVID-19, and the underlying disease remained stable: 531 patients with rheumatoid arthritis, 203 with psoriatic arthritis, 181 with spondyloarthropathies, and 40 with connective tissue diseases.

Not all authors suggest that antimalarial preparations may play a potential role in the prevention of infectious disease or that they could reduce its dynamics. Later works do not seem to confirm the prophylactic effect of antimalarial drugs against SARS-CoV-2 infection [41] or that treatment reduces the risk of hospitalization associated with the severe course of COVID-19 [42]. Today, it is believed that these drugs are not useful in the treatment of acute infections and have been withdrawn from the list of drugs recommended in COVID-19 therapy.

\section{Commentary}

Although CQ and HCQ are characterized by a similar mechanism of action, HCQ is more commonly used than CQ in chronic therapy in Western Europe and the USA. It is considered to have a better safety profile, to be better tolerated, and cause fewer side effects, especially in the gastrointestinal tract. It is the preferred preparation of the two, especially for children and pregnant women. The form of tablets, i.e. containing $400 \mathrm{mg}$ of the drug, seems to be more useful especially in the initial period of therapy, when higher daily doses are administered.

\section{Acknowledgments}

The study was conducted in the Department of Dermatology and Venereology, Medical University of Lodz, 90-647 Lodz, Plac Hallera 1 building 6.

\section{Conflict of interest}

The author declares no conflict of interest.

\section{References}

1. Rodriguez-Caruncho C, Bielsa Marsol I. Antimalarials in dermatology: mechanism of action, indications, and side effects. Actas Dermosifiliogr 2014; 105: 243-52.

2. Payne J. A postgraduate lecture on lupus erythematosus. Clin J 1894; 4: 223-9.

3. Woźniacka A, McCauliffe D. Optimal use of antimalarials in treating cutaneous lupus erythematosus. Am J Clin Dermatol 2006; 6: 1-11.

4. Ponticelli C, Moroni G. Hydroxychloroquine in systemic lupus erythematosus. Expert Opin Drug Saf 2017; 16: 411-9.

5. Mindell JA. Lysosomal acidification mechanisms. Annu Rev Physiol 2012; 74: 69-86.
6. Woźniacka A, Lesiak A, Boncela J, et al. The influence of antimalarial treatment on IL-1beta, IL- 6 and TNF-alpha mRNA expression on UVB-irradiated skin in systemic lupus erythematosus. Br I Dermatol 2008; 159: 1124-30.

7. Fujita Y, Matsuoka N, Temmoku J, et al. Hydroxychloroquine inhibits IL-1 $\beta$ production from amyloid-stimulated human neutrophils. Arthritis Res Ther 2019; 21: 250-8.

8. Chiche L, Jourde-Chiche N, Whalen E, et al. Modular transcriptional repertoire analyses of adults with systemic lupus erythematosus re-veal distinct type I and type II interferon signatures. Arthritis Rheumatol 2014; 66: 1583-95.

9. Niewold TB, Clark DN, Salloum R, et al. Interferon alpha in systemic lupus erythematosus. J Biomed Biotechnol 2010; 2010: 948364.

10. Moody KL, Uccellini MB, Avalos AM, et al. Toll-like receptordependent immune complex activation of B cells and dendrotic cells. Methods Mol Biol 2016; 1390: 249-72.

11. Garcia-Romo GS, Caielli S, Vega B, et al. Netting neutrophils are major inducers of type I IFN production in pediatric systemic lupus erythematosus. Sci Transl Med 2011; 3: 73ra20.

12. Torigoe M, Sakata K, Ishii A, et al. Hydroxychloroquine efficiently suppresses inflammatory responses of human classswitched memory B cells via Toll-like receptor 9 inhibition. Clin Immunol 2018; 195: 1-7.

13. Fox R. Anti-malarial drugs: possible mechanisms of action in autoimmune disease and prospects for drug development. Lupus 1996; 5 Suppl 1: S4-10.

14. Krutmann J, Grewe M. Involvement of cytokines, DNA damage, and reactive oxygen intermediates in ultraviolet radiation-induced modulation of intercellular adhesion molecule-1 expression. J Invest Dermatol 1995; 105: 67S-70S.

15. Shaffer B, Cahn MM, Levy EJ. Absorption of antimalarial drugs in human skin and corium. J Invest Dermatol 1951; 30: 341-5.

16. Danchenko N, Satia JA, Anthony MS. Epidemiology of systemic lupus erythematosus: a comparison of worldwide disease burden. Lupus 2006; 17: 1615-26.

17. Thomas G, Mancini J, Jourde-Chiche N. Mortality associated with systemic lupus erythematosus in France assessed by multiple-cause-of-death analysis. Arthritis Rheum 2014; 66: 2503-11.

18. Woźniacka A, Lesiak A, Smigielski J, et al. Wpływ leczenia fosforanem chlorochiny na parametry gospodarki lipidowej i wybrane wskaźniki laboratoryjne. Przegl Lek 2005; 62: 855-9.

19. Bili A, Sartorius JA, Kirchner JL, et al. Hydroksychloroquine use and decrease risk of diabetes in rheumatoid arthritis patients. J Clin Rheumatol 2011; 17: 115-20.

20. Morris SJ, Wasko MC, Antohe JL, et al. Hydroksychloroquine use associated with improvement in lipid profiles in rheumatoid arthritis patients. Arthritis Care Res 2011; 63: 530-4.

21. Rempenanault C, Combe B, Barnetche T, et al. Metabolic and cardiovascular benefits of hydroxychloroquine in patients with rheumatoid arthritis: a systematic review and metaanalysis. Ann Rheum Dis 2018; 77: 98-103.

22. Becker-Merok A, Nossent J. Prevalence, predictors and outcome of vascular damage in systemic lupus erythematosus. Lupus 2009; 18: 508-15.

23. Nikpour M, Urowitz MB, Ibanez D, et al. Importance of cumulative exposure to elevated cholesterol and blood pressurein development of atherosclerotic coronary artery disease in systemic lupus erythematosus: a prospective proof-of-concept cohort study. Arthritis Res Ther 2011; 13: R156.

24. Fasano S, Margiotta DP, Navarini L, et al. Primary prevention of cardiovascular disease in patients with systemic lupus 
erythematosus: case series and literature review. Lupus 2017; 26: 1463-72.

25. Pons-Estel GJ, Gonzalez LA, Zhang J, et al. Predictors of cardiovascular damage in patients with systemic lupus erythematosus: data from LUMINA (LXVIII), a multiethnic US cohort. Rheumatology 2009; 48: 817-22.

26. Liu D, Li X, Zhang Y, et al. Chloroquine and hydroxychloroquine are associated with reduced cardiovascular risk: a systematic review and meta-analysis. Drug Des Devel Ther 2018; 12: 1685-95.

27. Tselios K, Gladman DD, Touma Z, et al. Disease course patterns in systemic lupus erythematosus. Lupus 2019; 28: 114-22.

28. Ruiz-Irastorza G, Martin-Iglesias D, Soto-Peleteiro A. Update on antimalarials and systemic lupus erythematosus. Curr Opin Rheumatol 2020; 32: 572-82.

29. Garg S, Unnithan R, Hansen KE, et al. The clinical significance of monitoring hydroxychloroquine levels in patients with systemic lupus erythematosus: a systematic review and meta-analysis. Arthritis Care Res 2021; 73: 707-16.

30. Mehat P, Atiquzzaman M, Esdaile JM, et al. Medication nonadherence in systemic lupus erythematosus: a systematic review. Arthritis Care Res 2017; 69: 1706-13.

31. Martin-Iglesias D, Artaraz J, Fonollosa A, et al. Evolution of retinal changes measured by optical coherence tomography in the assessment of hydroksychloroquine ocular safety in patients with systemic lupus erythematosus. Lupus 2019; 28: 555-9.

32. Mukwikiwi ER, Pineau CA, Vinet E, et al. Retinal complications in patients with systemic lupus erythematosus treated with antimalarial drugs. J Rheumatol 2020; 47: 553-6.

33. Ezra N, Jorizzo J. Hydroxychloroquine and smoking in patients with cutaneous lupus erythematosus. Clin Exp Dermatol 2012; 37: 327-34.

34. Woźniacka A, McCauliffe DP. Optimal use of antimalarials in treating cutaneous lupus erythematosus. Am J Clin Dermatol 2006; 6: 1-11.

35. Finielz P, Gendoo Z, Chuel C, Guiserix J. Interaction between cyclosporin and chloroquine. Nephron 1993; 65: 333.

36. Buchanan NM, Toubi E, Khamashta M, et al. Hydroxychloroquine and lupus pregnancy: review of a series of 36 cases. Ann Rheum Dis 1996; 55: 486-8.

37. Ulberg S, Linquist NG, Sjostrand SE. Accumulation of chorioretinotoxic drugs in the fetal eye. Nature 1970; 227: 1257-8.

38. Barsalou J, Jaeggi E, Laskin CA, et al. Prenatal exposure to antimalarials decreases the risk of cardiac but not non-cardiac neonatal lupus: a single-centre cohort study. Rheumatology 2017; 56: 1552-9.

39. Meyerowitz EA, Vannier AGL, Friesen MGN, et al. Rethinking the role of hydroxychloroquine in the treatment of COVID-19. FASEB I 2020; 34: 6027-37.

40. Favalli EG, Monti S, Ingegnoli F, et al. Incidence of COVID-19 in patients with rheumatic diseases treated with targeted immunosuppressive drugs: what can we learn from observational data. Arthritis Rheum 2020; 72: 1600-6.

41. Favalli EG, Agape E, Caporali R. Incidence and clinical course of COVID-19 in patients with connective tissue diseases: a descriptive observational analysis. J Rheumatol 2020; 47: 1296.

42. Gianfrancesco M, Hyrich KL, Al-Adely S, et al. Characteristics associated with hospitalisation for COVID-19 in people with rheumatic disease: data from the COVID-19 global rheumatology alliance physician-reported registry. Ann Rheum Dis 2020; 79: 859-66. 